\title{
A Case for Redundant Arrays of Inexpensive Disks (RAID)
}

\author{
Daved A Patterson, Garth Gibson, and Randy $H$ Katz \\ Computer Science Division \\ Department of Electrical Enguneering and Computer Sciences \\ 571 Evans Hall \\ University of Californua \\ Berkeley, CA 94720 \\ (pattrsn@gunger berkeley edu)
}

\begin{abstract}
Increasing performance of CPUs and memories will be squandered if not matched by a sumular performance increase in IIO While the capactry of Single Large Expensive Disks (SLED) has grown rapidly. the performance improvement of SLED has been modest Redundant Arrays of Inexpensive Disks (RAID), based on the magnetic disk technology developed for personal computers, offers an attractuve alternative to SLED, promising improvements of an order of magnitude in performance, relability, power consumption, and scalability This paper introduces five levels of RAIDs, giving their relative cost/performance, and compares RAID to an IBM 3380 and a Fujtsu Super Eagle
\end{abstract}

\section{Background: Rising CPU and Memory Performance}

The users of computers are currently enjoying unprecedented growth in the speed of computers Gordon Bell sard that between 1974 and 1984, single chip computers improved in performance by $40 \%$ per year, about twice the rate of minicomputers [Bell 84] In the following year Bill Joy predicted an even faster growth [Joy 85]

$$
M I P S=2^{\text {Year }-1984}
$$

Mainframe and supercomputer manufacturers, having difficulty keeping pace with the rapid growth predicted by "Joy's Law," cope by offerng multuprocessors as therr top-of-the-line product.

But a fast CPU does not a fast system make Gene Amdahl related CPU speed to main memory size using this rule [S1ewiorek 82]

Each CPU instruction per second requures one byte of matn memary,

If computer system costs are not to be dominated by the cost of memory, then Amdahl's constant suggests that memory chip capacity should grow at the same rate Gordon Moore predicted that growth rate over 20 years ago

$$
\text { transestors/chp }=2^{\text {Year }-1964}
$$

As predicted by Moore's Law, RAMs have quadrupled in capacity every two [Moore 75] to three years [Myers 86]

Recently the ratio of megabytes of main memory to MIPS has been defined as alpha [Garcla 84], with Amdahl's constant meaning alpha = 1 In part because of the rapid drop of memory prices, main memory sizes have grown faster than CPU speeds and many machunes are shipped today with alphas of 3 or higher

To maintain the balance of costs in computer systems, secondary storage must match the advances in other parts of the system A key meas-

Permission to copy without fee all or part of this material is granted provided that the copies are not made or distributed for direct commercial advantage, the ACM copynight notice and the title of the publication and its da: appear, and notice is given that copying is by permussion of the Association for Computing Machinery To copy otherwise, or to republish, requires a fee and / or specific permission

(C) 1988 ACM 0-89791-268-3/88/0006/0109\$150 ure of magneuc disk technology is the growth in the maximum number of bits that can be stored per square inch, or the bits per inch in a track tumes the number of tracks per inch Called MA D, for maximal areal density, the "First Law in Disk Density" predicts [Frank87]

$$
M A D=10^{(\text {Year }-1971) / 10}
$$

Magnetic disk technology has doubled capacity and halved price every three years, in line with the growth rate of semiconductor memory, and in practice between 1967 and 1979 the disk capacity of the average IBM data processing system more than kept up with its main memory [Stevens81]

Capacity is not the ouly memory characteristic that must grow rapidly to maintain system balance, since the speed with which instructions and data are delivered to a CPU also determines its ulumatc performance The speed of man memory has kept pace for two reasons

(1) the invention of caches, showing that a small buffer can be managed automancally to contain a substantial fraction of memory references,

(2) and the SRAM technology, used to build caches, whose speed has improved at the rate of $40 \%$ to $100 \%$ per year

In contrast to primary memory technologies, the performance of single large expensive magnetic disks (SLED) has improved at a modest rate These mechanical devices arc dominated by the seek and the rotation delays from 1971 to 1981 , the raw seek tume for a high-end IBM disk improved by only a factor of two while the rotation ume did not change[Harker81] Greater density means a higher transfer rate when the information is found, and extra heads can reduce the average seek tume, but the raw seek tume only improved at a rate of $7 \%$ per year There is no reason to expect a faster rate in the near future

To maintain balance, computer systems have been using even larger main memories or solid state disks to buffer some of the $\mathrm{I} / \mathrm{O}$ activity This may be a fine solution for applications whose I/O activity has locality of reference and for which volatility is not an 1ssue, but applications dominated by a high rate of random requests for small pieces of data (such as transaction-processing) or by a low number of requests for massive amounts of data (such as large simulations running on supercomputers) are facing a senous performance limitation

\section{The Pending I/O Crisis}

What is the impact of improving the performance of some pieces of a problem while leaving others the same? Amdahl's answer is now known as Amdahl's Law [Amdahl67]

$$
S=\frac{1}{(1-f)+f / k}
$$

where

$S=$ the effectuve speedup.

$f=$ fraction of work in faster mode, and

$k=$ speedup while in faster mode

Suppose that some current applications spend $10 \%$ of therr ume in I/O Then when computers are $10 X$ faster--according to Bill Joy in just over three years--then Amdahl's Law predicts effective speedup will be only $5 \mathrm{X}$ When we have computers $100 \mathrm{X}$ faster--via evolution of uniprocessors or by multiprocessors--this application will be less than 10X faster, wastung $90 \%$ of the potentual speedup 
While we can imagine improvements in software file systems via buffering for near term $1 / O$ demands, wc nced innovation to avoid an $1 / O$ crisis [Boral 83]

\section{A Solution: Arrays of Inexpensive Disks}

Rapid improvements in capacity of large disks have not been the only target of disk designers, since personal computers have created a market for inexpensive magnetic disks These lower cost disks have lower performance as well as less capacity Table I below compares the top-of-the-line IBM 3380 model AK4 manframe disk, Fujıtsu M2361A "Super Eagle" minicomputer disk, and the Conner Penpherals CP 3100 personal computer disk

\begin{tabular}{|c|c|c|c|c|c|}
\hline Characteristics & $\begin{array}{l}I B M \\
3380\end{array}$ & $\begin{array}{l}\text { Fujtsu } \\
\text { M2361A }\end{array}$ & $\begin{array}{l}\text { Conners } \\
\text { CP3100 }\end{array}$ & \multicolumn{2}{|c|}{$\begin{array}{l}3380 \vee 2361 v \\
31003100 \\
(>1 \text { means } \\
3100 \text { as better })\end{array}$} \\
\hline Disk duameter (unches) & 14 & 105 & 35 & 4 & 3 \\
\hline Formatted Data Capacity (MB) & 7500 & 600 & 100 & & 012 \\
\hline Price/MB(controller incl) & $\$ 18-\$ 10$ & $\$ 20-\$ 17 \$ 1$ & $0-\$ 7$ & $1-25$ & $5 \quad 17.3$ \\
\hline MTTF Rated (hours) & 30,000 & 20,00030 &, 000 & 1 & 15 \\
\hline MTTF in practuce (hours) & 100,000 & $?$ & $?$ & $?$ & $?$ \\
\hline No Actuators & 4 & 1 & 1 & & 21 \\
\hline Maximum I/O's/second/Actuato & 50 & 40 & 30 & & 68 \\
\hline Typical I/O's/second/Actuator & 30 & 24 & 20 & & 8 \\
\hline Maximum L/O's/second/box & 200 & 40 & 30 & & 8 \\
\hline Typical I/O's/second/box & 120 & 24 & 20 & & 8 \\
\hline Transfer Rate (MB/sec) & 3 & 25 & 1 & & 4 \\
\hline Power/box (W) & 6,600 & 640 & 10 & 660 & 64 \\
\hline Volume (cu ft) & 24 & 34 & 03 & 800 & 110 \\
\hline
\end{tabular}

Table I Comparison of IBM 3380 disk model AK4 for mainframe computers, the Funtsu M2361A "Super Eagle" disk for mucomputers, and the Conners Peripherals CP 3100 disk for personal computers By "Maximum IIO's/second" we mean the maximum number of average seeks and average rotates for a single sector access Cost and reliability information on the 3380 comes from widespread expernence [IBM 87] [Gawlick87] and the information on the Fujusu from the manual [Funtsu 87]. while some numbers on the new CP3100 are based on speculation The price per megabyte is given as a range to allow for different pnces for volume discount and different mark-up practices of the vendors (The 8 walt maximum power of the CP3100 was increased to 10 watts to allow for the inefficiency of an external power supply, since the other drives contain their own power supplies)

One surprising fact is that the number of $1 / O$ s per second per actuator in an inexpensive disk is within a factor of two of the large disks In several of the remaining metrics, including price per megabyte, the inexpensive disk is superior or equal to the large disks

The small size and low power are even more impressive since disks such as the CP3100 contain full track buffers and most functions of the traditional mainframe controller Small disk manufacturers can provide such functions in high volume disks because of the efforts of standards committees in defining hugher level peripheral interfaces, such as the ANSI X3 131-1986 Small Computer System Interface (SCSI) Such standards have encouraged companies like Adeptec to offer SCSI interfaces as single chips, in turn allowing disk companies to embed mainframe controller functions at low cost Figure 1 compares the traditional mainframe disk approach and the small computer disk approach The same SCSI interface chip embedded as a controller in every disk can also be used as the durect memory access (DMA) device at the other end of the SCSI bus

Such characteristics lead to our proposal for building $1 / O$ systems as arrays of inexpensive disks, either interleaved for the large transfers of supercomputers [Kim 86][Livny 87][Salem86] or independent for the many small transfers of transaction processing Using the information in Table 1,75 inexpensive disks potentially have 12 tmes the $\mathrm{I} / \mathrm{O}$ bandwidth of the IBM 3380 and the same capacity, with lower power consumption and cost

\section{Caveats}

We cannot explore all issues associated with such arrays in the space avaliable for this paper, so we concentrate on fundamental estimates of price-performance and reliability Our reasoning is that if there are no advantages in pnce-performance or temble disadvantages in reliability, then there is no need to explore further We characterize a transaction-processing workload to evaluate performance of a collection of inexpensive disks, but remember that such a collection is just one hardware component of a complete tranaction-processing system While designing a complete TPS based on these ideas is enticing, we will resist that temptation in this paper Cabling and packaging, certainly an issue in the cost and reliability of an array of many inexpensive disks, is also beyond this paper's scope

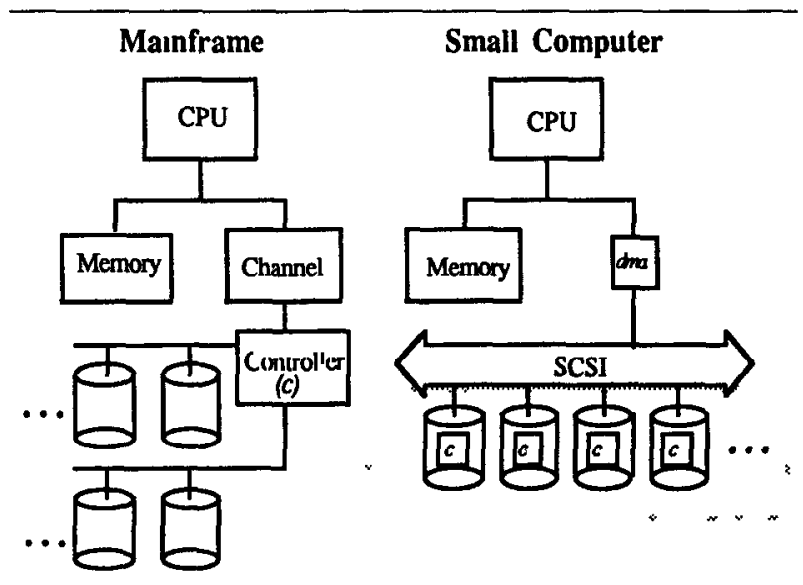

Figure 1 Comparison of organtzations for typical mainframe and small computer disk interfaces Single chip SCSI interfaces such as the Adaptec AIC-6250 allow the small computer to use a single chup to be the DMA interface as well as provide an embedded controller for each dusk /Adeptec 87] (The price per megabyte in Table I includes everything in the shaded boxes above)

\section{And Now The Bad News: Reliability}

The unreliability of disks forces computer systems managers to make backup versions of information quite frequently in case of fallure What would be the impact on reliability of having a hundredfold increase in disks? Assuming a constant failure rate--that is, an exponentially distributed time to failure--and that failures are independent--both assumptions made by disk manufacturers when calculatung the Mean Time To Fallure (MTTF)--the reliability of an array of disks is

$$
\text { MTTF of a Disk Array }=\frac{M T T F \text { of a Single Dusk }}{\text { Number of Disks in the Array }}
$$

Using the information in Table I, the MTTF of 100 CP 3100 disks is $30,000 / 100=300$ hours, or less than 2 weeks Compared to the 30,000 hour (> 3 years) MTTF of the IBM 3380, this is dismal If we consider scalıng the array to 1000 disks, then the MTTF is 30 hours or about one day, requinng an adjectuve worse than dismal

Without fault tolerance, large arrays of inexpensive disks are too unreliable to be useful

\section{A Better Solution- RAID}

To overcome the reliability challenge, we must make use of extra disks containing redundant informauon to recover the original information when a disk farls Our acronym for these Redundant Arrays of Inexpensive Disks is RAID To simplify the explanation of our final proposal and to avoid confusion with previous work, we give a taxonomy of five different organizations of disk arrays, beginning with mirrored disks and progressing through a vanety of alternatives with diffening performance and reliability We refer to each organization as a RAID level

The reader should be forewarned that we describe all levels as if implemented in hardware solely to simplify the presentation, for RAID 1deas are applicable to software implementations as well as hardware

Reliability Our basic approach will be to break the arrays into reliability groups, with each group having extra "check" disks containing redundant information When a disk fails we assume that within a short time the failed disk will be replaced and the information will be 
reconsuluted on to the new disk using the redundant information This tume is culled the mean ume to repair (MTTR) The MTTR can be reduced If the system includes extra disks to act as "hot" standby spares, when a disk fails, a replacement disk is switched in electronically Penodically a human operator replaces all falled disks Here are other terms that we use

$D=$ total number of disks with data (not including extra check disks),

$G=$ number of data disks in a group (not including extra check disks),

$C=$ number of check disks in a group,

$n_{G}=D / G=$ number of groups,

As mentroned above we make the same assumptions that disk manufacturers make--that fallures are exponentual and independent (An earthquake or power surge is a sttuation where an array of disks might not fall independently) Since these reltability predictions will be very high, we want to emphasize that the reliability is only of the the disk-head assemblies with this failure model, and not the whole software and electronic system In addition, in our view the pace of technology means extremely high MTTF are "overkıll"--for, independent of expected lifeume, users will replace obsolete disks After all, how many people are still using 20 year old disks?

The general MTTF calculation for single-error reparing RAID is given in two steps First, the group MTTF is

$$
M T T F_{\text {Group }}=\frac{M T T F_{\text {Dusk }}}{G+C} * \frac{1}{\begin{array}{c}
\text { Probabilty of another falure in a group } \\
\text { before repainng the dead disk }
\end{array}}
$$

As more formally denved in the appendix, the probabiluty of a second fallure before the first has been repaured is

$$
\begin{aligned}
& \text { Probability of } \\
& \text { Another Fallure }
\end{aligned}=\frac{M T T R}{M T T F_{D_{\text {tsk }}} /(\text { No Disks-1) }}=\frac{M T T R}{M T T F_{D_{\text {Lsk }}} /(G+C-1)}
$$

The inttition behind the formal calculation in the appendix comes from trying to calculate the average number of second disk fallures during the repair tume for $X$ single disk fallures Since we assume that disk falures occur at a uniform rate, this average number of second fallures durng the reparr tume for $X$ first fallures is

\section{$X * M T T R$}

$$
\text { MTTF of remaineng disks in the group }
$$

The average number of second failures for a single disk is then

$$
\text { MTTR }
$$

$M T T F_{\text {Disk }} /$ No of remaining disks in the group

The MTTF of the remaining disks is just the MTTF of a single disk divided by the number of good disks in the group, giving the result above

The second step is the reliability of the whole system, which is approximately (since $M T T F_{\text {Group }}$ is not quite distrubuted exponentally)

$M T T F_{\text {RAID }}=\frac{M T T F_{\text {Group }}}{n_{G}}$

Plugging it all together, we get.

$$
\begin{aligned}
M T T F_{R A I D} & =\frac{M T T F_{D i s k}}{G+C} * \frac{M T T F_{D i s k}}{(G+C-1)^{*} M T T R} * \frac{1}{n_{G}} \\
& =\frac{\left(M T T F_{D i s k}\right)^{2}}{(G+C)^{*} n_{G} *(G+C-1)^{*} M T T R} \\
M T T F_{R A I D} & =\frac{\left(M T T F_{D i s k}\right)^{2}}{\left(D+C^{*} n_{G}\right)^{*}(G+C-1)^{*} M T T R}
\end{aligned}
$$

Since the formula is the same for each level, we make the abstract numbers concrete using these parameters as appropriate $D=100$ total data disks, $G=10$ data disks per group, $M T T F_{D_{l s k}}=30,000$ hours, $M T T R=1$ hour, with the check disks per group $C$ determined by the RAID level

Reliability Overhead Cost This is simply the extra check disks, expressed as a percentage of the number of data disks $D$ As we shall see below, the cost vanes with RAID level from $100 \%$ down to $4 \%$

Useable Storage Capacity Percentage Another way to express this reliability overhead is in terms of the percentage of the total capacity of data disks and check disks that can be used to store data Depending on the organization, this varies from a low of $50 \%$ to a high of 96\%

Performance Since supercomputer applications and transaction-processing systems have different access patterns and rates, we need different metrics to evaluate both For supercomputers we count the number of reads and writes per second for large blocks of data, with large defined as getung at least one sector from each data disk in a group Durng large transfers all the disks in a group act as a single unit, each reading or writing a portion of the large data block in parallel

A better measure for transaction-processing systems is the number of individual reads or writes per second Since transaction-processing systems (e g, debits/credits) use a read-modify-write sequence of disk accesses, we include that metric as well Ideally dunng small transfers each disk in a group can act independently, etther reading or wntung independent information In summary supercomputer applications need a high data rate while transaction-processing need a high $1 / O$ rate

For both the large and small transfer calculations we assume the minimum user request is a sector, that a sector is small relative to a track, and that there is enough work to keep every device busy Thus sector size affects both disk storage efficiency and transfer size Figure 2 shows the ideal operation of large and small disk accesses in a RAID

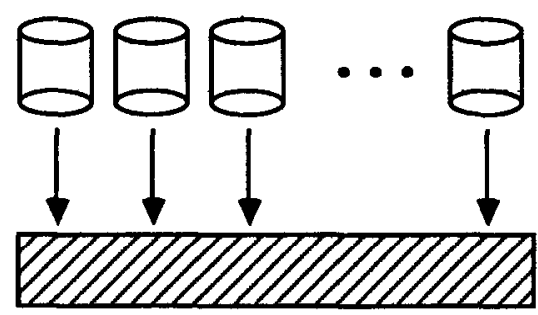

(a) Single Large or "Grouped" Read (1 read spread over $G$ dusks)

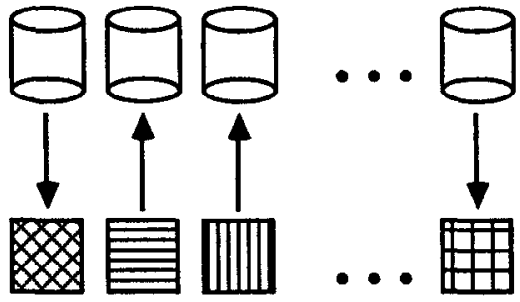

(b) Several Small or Individual Reads and Wrutes ( $G$ reads and/or wrtes spread over $G$ disks)

Figure 2. Large transfer vs small transfers in a group of $G$ disks

The six performance metrics are then the number of reads, writes, and read-modify-writes per second for both large (grouped) or small (individual) transfers Rather than give absolute numbers for each metric, we calculate efficiency the number of events per second for a RAID relative to the corresponding events per second for a single disk (This is Boral's I/O bandwidth per gigabyte [Boral 83] scaled to gigabytes per disk) In this paper we are after fundamental differences so we use simple, determinisuc throughput measures for our performance metric rather than latency

Effective Performance Per Disk The cost of disks can be a large portion of the cost of a database system, so the 1/O performance per disk--factoring in the overhead of the check disks--suggests the cost/performance of a system This is the bottom line for a RAID 


\section{First Level RAID: Mirrored Disks}

Mirrored disks are a tradıtional approach for improving reliability of magnetic disks This is the most expensive option we consider since all disks are duplicated ( $G=1$ and $C=1$ ), and every write to a data disk is also a write to a check disk Tandem doubles the number of controllers for fault tolerance, allowing an opumized version of mirrored disks that lets reads occur in parallel Table II shows the metrics for a Level 1 RAID assuming this optimization

\begin{tabular}{|c|c|c|}
\hline$M T T F$ & \multicolumn{2}{|c|}{$\begin{array}{l}\text { Exceeds Useful Product Lufeume } \\
(4,500,000 \text { hrs or }>500 \text { years })\end{array}$} \\
\hline Total Number of Disks & 2D & \\
\hline Overhead Cost & $100 \%$ & \\
\hline Useable Storage Capactty & $50 \%$ & \\
\hline Events/Sec vs Single Disk & Full RAID & Efficiency Per Disk \\
\hline $\begin{array}{l}\text { Large (or Grouped) Reads } \\
\text { Large (or Grouped) Writes }\end{array}$ & $\begin{array}{l}2 D / S \\
D / S\end{array}$ & $\begin{array}{r}100 / \mathrm{S} \\
50 / \mathrm{S}\end{array}$ \\
\hline Large (or Grouped) $R \cdot M-W$ & $4 D / 3 S$ & $67 / S$ \\
\hline Small (or Individual) Reads & $2 D$ & 100 \\
\hline Small (or Individual) Writes & $D$ & 50 \\
\hline Small (or Individual) $R-M-W$ & $4 D / 3$ & 67 \\
\hline
\end{tabular}

Table II. Characteristics of Level I RAJD Here we assume that writes are not slowed by walting for the second write to complete because the slowdown for writing 2 disks is minor compared to the slowdown $S$ for wrtting a whole group of 10 to 25 disks Unlike a "pure" murrored scheme with extra disks that are invisible to the software, we assume an optumuzed scheme vith twice as many çontrollers allowing parallel reads to all disks, giving full disk bandwidth for large reads and allowing the reads of read-modify-writes to occur in parallel

When individual accesses are distributed across multuple disks, average queueing, seek, and rotate delays may differ from the single disk case Although bandwidth may be unchanged, it is distributed more evenly, reducing variance in queueing delay and, if the disk load is not too high, also reducing the expected queueing delay through parallelism [Livny 87] When many arms seek to the same track then rotate to the described sector, the average seek and rotate tume will be larger than the average for a single disk, tending toward the worst case tumes This affect should not generally more than double the average access tume to a single sector while still getting many sectors in parallel In the special case of mirrored disks with sufficient controllers, the choice between arms that can read any data sector will reduce the tume for the average read seek by up to $45 \%$ [Bitton 88]

To allow for these factors but to retain our fundamental emphasis we apply a slowdown factor, $S$, when there are more than two disks in a group In general, $1 \leq S \leq 2$ whenever groups of disk work in parallel With synchronous disks the spindles of all disks in the group are synchronous so that the corresponding sectors of a group of disks pass under the heads simultaneously,[Kurzwell 88] so for synchronous disks there is no slowdown and $S=1$ Since a Level 1 RAID has only one data disk in its group, we assume that the large transfer requires the same number of disks acting in concert is found in groups of the higher level RAIDs 10 to 25 disks

Duplicating all disks can mean doubling the cost of the database system or using only $50 \%$ of the disk storage capacity Such largess inspires the next levels of RAID

\section{Second Level RAID: Hamming Code for ECC}

The history of main memory organizations suggests a way to reduce the cost of reliability $W_{1}$ th the introduction of $4 \mathrm{~K}$ and $16 \mathrm{~K}$ DRAMs, computer designers discovered that these new devices were subject to losing information due to alpha particles Since there were many single bit DRAMs in a system and since they were usually accessed in groups of 16 to 64 chips at a time, system designers added redundant chips to correct single errors and to detect double errors in each group This increased the number of memory chips by $12 \%$ to $38 \%$-depending on the size of the group--but it significantly improved reliability

As long as all the data bits in a group are read or written together, there is no impact on performance However, reads of less than the group stze require reading the whole group to be sure the informaticn is correct, and writes to a portuon of the group mean three steps
1) a read step to get all the rest of the data,

2) a modify step to merge the new and old information

3) a write step to write the full group, including check information

Since we have scores of disks in a RAID and since some accesses are to groups of disks, we can mimic the DRAM solution by bit-interleaving the data across the disks of a group and then add enough check disks to detect and correct a single error A single parity disk can detect a single error, but to correct an error we need enough check disks to identufy the disk with the error For a group size of 10 data disks $(G)$ we need 4 check disks $(C)$ in total, and if $G=25$ then $C=5$ [Hamming50] To keep down the cost of redundancy, we assume the group size will vary from 10 to 25

Since our individual data transfer unit is just a sector, bit- interleaved disks mean that a large transfer for this RAID must be at least $G$ sectors Like DRAMs, reads to a smaller amount implies reading a full soctor from each of the bit-interleaved disks in a group, and writes of a single unit involve the read-modify-wnte cycle to all the disks Table III shows the metrics of this Level 2 RAID

\begin{tabular}{|c|c|c|c|c|c|}
\hline \multirow{2}{*}{\multicolumn{2}{|c|}{$M T T F$}} & \multicolumn{4}{|c|}{ Exceeds Useful Lifetume } \\
\hline & & \multicolumn{2}{|c|}{$\begin{array}{l}G=10 \\
(494,500 \text { hrs } \\
\text { or }>50 \text { years })\end{array}$} & \multicolumn{2}{|c|}{$\begin{array}{l}G=25 \\
(103,500 \text { hrs } \\
\text { or } 12 \text { years })\end{array}$} \\
\hline \multicolumn{2}{|c|}{ Total Number of Disks } & \multicolumn{2}{|c|}{1400} & \multicolumn{2}{|c|}{$1.20 \mathrm{D}$} \\
\hline \multirow{2}{*}{\multicolumn{2}{|c|}{$\begin{array}{l}\text { Overhead Cast } \\
\text { Useable Storage Capactsy }\end{array}$}} & \multicolumn{2}{|c|}{$40 \%$} & \multicolumn{2}{|l|}{$20 \%$} \\
\hline & & \multirow{2}{*}{\multicolumn{2}{|c|}{$\begin{array}{l}71 \% \\
\text { Efficlency Per Disk }\end{array}$}} & \multicolumn{2}{|l|}{$83 \%$} \\
\hline Events/Sec & Full RAID & & & Efficte & ncy Per Dusk \\
\hline (vs Single Disk) & & $L 2$ & $L 2 / L 1$ & $L 2$ & $L 2 / L 1$ \\
\hline Large Reads & $D / S$ & $71 / \mathrm{s}$ & $71 \%$ & $86 / S$ & $86 \%$ \\
\hline Large Writes & $D / S$ & $71 / \mathrm{S}$ & $143 \%$ & $86 / \mathrm{S}$ & $172 \%$ \\
\hline Large $R-M-W$ & $D / S$ & $71 / \mathrm{S}$ & $107 \%$ & $86 / S$ & $129 \%$ \\
\hline Small Reads & $D / S G$ & $07 / \mathrm{s}$ & $6 \%$ & $03 / \mathrm{S}$ & $3 \%$ \\
\hline Small Writes & $D / 2 S G$ & $04 / \mathrm{S}$ & $6 \%$ & $02 / \mathrm{S}$ & $3 \%$ \\
\hline Small $R-M-W$ & $D / S G$ & $07 / \mathrm{S}$ & 9\% & $03 / \mathrm{S}$ & $4 \%$ \\
\hline
\end{tabular}

Table III Characteristics of a Level 2 RAID The L2/LI column gives the \% performance of level 2 in terms of level 1 (>100\% means $L 2$ is faster) As long as the transfer unt is large enough to spread over all the data disks of a group, the large IIOs get the full bandwidth of each disk, divided by $S$ to allow all disks in a group to complete Level 1 large reads are faster because data is duplicated and so the redundancy disks can also do independent accesses Small IIOs still require accessing all the disks in a group, so only D/G small I/O can happen at a time, again divided by $S$ to allow a group of disks to finish Small Level 2 writes are like small $R-M-W$ because full sectors must be read before new data can be written onto part of each sector

For large writes, the level 2 system has the same performance as level 1 even though it uses fewer check disks, and so on a per disk basis it outperforms level 1 For small data transfers the performance is dismal either for the whole system or per disk, all the disks of a group must be accessed for a small transfer, limiting the paximum number of simultaneous accesses to $D / G$ We also include the slowdown factor $S$ since the access must watt for all the disks to complete

Thus level 2 RAID is desirable for supercomputers but inappropriate for transaction processing systems, with increasing group size increasing the disparity in performance per disk for the two applications In recognition of this fact, Thinking Machines Incorporated announced a Level 2 RAID this year for its Connection Machine supercomputer called the "Data Vault," with $G=32$ and $C=8$, including one hot standby spare [Hillis 87]

Before improving small data transfers, we concentrate once more on lowering the cost

\section{Third Level RAID: Single Check Disk Per Group}

Most check disks in the level 2 RAID are used to determine which disk failed, for only one redundant parity disk is needed to detect an error These extra disks are truly "redundant" since most disk controllers can already detect if a disk fauled ether through special signals provided in the disk interface or the extra checking informauon at the end of a sector used to detect and correct soft errors So information on the falled disk can be reconstructed by calculatung the parity of the remaining good disks and then companing bit-by-bit to the panty calculated for the onginal full 
group When these two partics agree, the fauled bit was a 0 , otherwise it was a 1 If the check disk is the failure, just read all the data disks and store the group panty in the replacement disk

Reducing the check disks to one per group $(C=1)$ reduces the overhead cost to between $4 \%$ and $10 \%$ for the group sizes considered here The performance for the third level RAID system is the same as the Level 2 RAID, but the effective performance per disk increases since it needs fewer chcck disks This reduction in total disks also increases reliability, but since it is still larger than the useful lifetime of disks, this is a minor point One advantage of a level 2 system over level 3 is that the extra check information associated with each sector to correct soft errors ts not needed, increasing the capacity per disk by perhaps $10 \%$ Level 2 also allows all soft errors to be corrected "on the fly" without having to reread a sector Table IV summanzes the third level RAID characteristics and Figure 3 compares the sector layout and check disks for levels 2 and 3

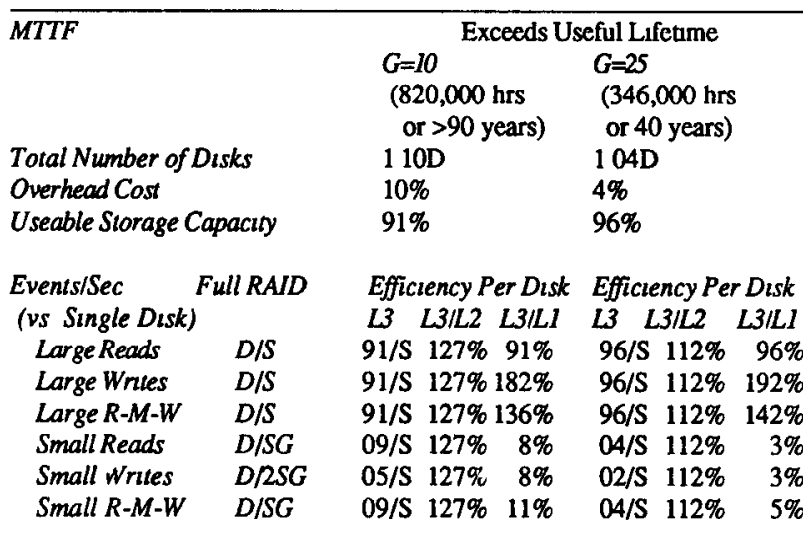

Table IV Characteristics of a Level 3 RAID The L3/L2 column gives the \% performance of $L 3$ in terms of $L 2$ and the $L 3 / L 1$ column gives $u$ in terms of $L I$ ( $>100 \%$ means $L 3$ is faster) The performance for the full systems is the same in RAID levels 2 and 3, but since there are fewer check disks the performance per disk improves

Park and Balasubramanian proposed a third level RAID system without suggesting a particular application [Park86] Our calculations suggest it is a much better match to supercomputer applications than to transaction processing systems This year two disk manufacturers have announced level 3 RAIDs for such applications using synchronized 525 inch disks with $\mathrm{G}=4$ and $\mathrm{C}=1$ one from Maxtor and one from Micropolis [Maginnis 87]

This third level has brought the reliability overhead cost to its lowest level, so in the last two levels we improve performance of small accesses without changing cost or reliability

\section{Fourth Level RAID Independent Reads/Writes}

Spreading a transfer across all disks within the group has the following advantage

- Large or grouped transfer tume is reduced because transfer bandwidth of the enture array can be explotted.

But it has the following disadvantages as well

- Reading/writung to a disk in a group requires reading/wntung to all the disks in a group, levels 2 and 3 RAIDs can perform only one $\mathrm{I} / \mathrm{O}$ at a tume per group

- If the disks are not synchronized, you do not see average seek and rotational delays, the observed delays should move towards the worst case, hence the $S$ factor in the equations above

This fourth level RAID improves performance of small transfers through parallelism--the ability to to more than one I/O per group at a time We no longer spread the individual transfer information across several disks, but keep each individual unit in a single disk

The virtue of bit-interleaving is the easy calculation of the Hamming code needed to detect or correct errors in level 2 But recall that in the third level RAID we rely on the disk controller to detect errors within a single disk sector Hence, if we store an individual transfer unit in a single sector, we can detect errors on an individual read without accessing any other disk Figure 3 shows the different ways the information is stored in a sector for
RAID levels 2, 3, and 4 By storing a whole transfer unit in a sector, reads can be independent and operate at the maximum rate of a disk yet stıll detect errors Thus the primary change between level 3 and 4 is that we interleave data between disks at the secwor level rather than at the bit level

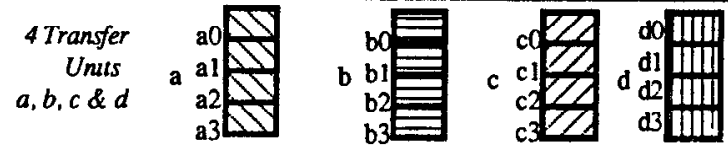

Level 2 Level 3 Level 4

$\begin{array}{cccc}\text { Sector } 0 & \\ \text { Data } & \text { bisk 1 }\end{array}$

Figure 3 Comparison of location of data and check information in sectors for RAID levels 2, 3, and 4 for $G=4$ Not shown is the small amount of check information per sector added by the disk controller to detect and correct soft errors within a sector Remember that we use physical sector numbers and hardware control to explain these ideas but $R A J D$ can be implemented by software using logical sectors and disks

At first thought you might expect that an individual write to a singls sector still involves all the disks in a group since (1) the check disk must be rewritten with the new panty data, and (2) the rest of the data disks must be read to be able to calculate the new panty data Recall that each parity bit is just a single exclusive OR of all the corresponding data bit in a group In level 4 RAID, unlike level 3, the parity calculation is mich simpler since, if we know the old data value and the old parity value as well as the new data value, we can calculate the new parity information as follows

$$
\text { new party }=\text { (old data xor new data }) \text { xor old parit }
$$

In level 4 a small write then uses 2 disks to perform 4 accesses-- 2 reads and 2 wntes--while a small read involves only one read on one disk Table $\mathrm{V}$ summarizes the fourth level RAID charactenstics Nore that all small accesses improve--dramatically for the reads--but the small read-modify-write is still so slow relative to a level I RAID that its applicability to transaction processing is doubtful Recently Salem and Garcia-Molina proposed a Level 4 system [Salem 86]

Before proceeding to the next level we need to explain the performance of small writes in Table $V$ (and hence small read-modify-writes since they ental the same operations in this RAID) The formula for the small writes divides $D$ by 2 instead of 4 becausi ? 
accesses can proceed in parallel the old data and old parity can be read at the same tume and the new data and new parity can be wniten at the same time The performance of small writes is also divided by $G$ because the single check disk in a group must be read and written with every small write in that group, thereby limitung the number of writes that can be performed at a tume to the number of groups

The check disk is the bottleneck, and the final level RAID removes this bottleneck

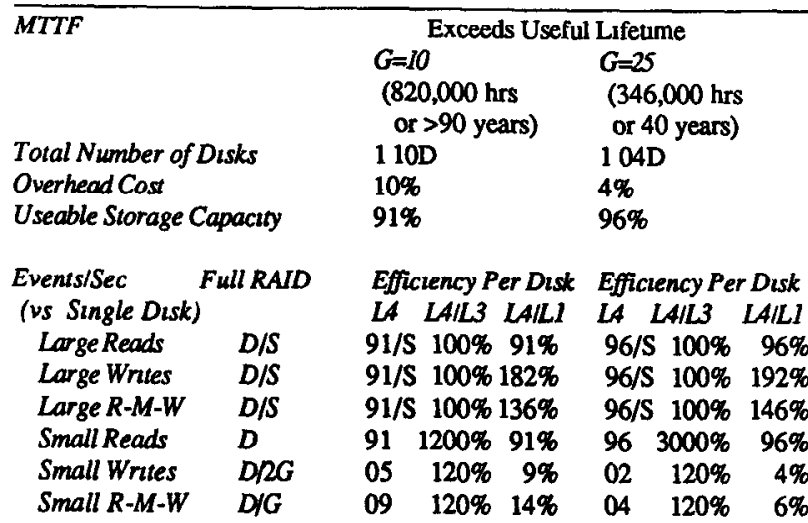

Table V. Characteristics of a Level 4 RAID The LAIL3 column gives the \% performance of $L A$ in terms of $L 3$ and the $L A I L I$ column gives $t$ in terms of $L 1$ ( $>100 \%$ means $L A$ is faster) Small reads improve because they no longer the up a whole group at a tume Small wrtes and $R-M-W S$ improve some because we make the same assumptions as we made in Table II the slowdown for two related IIOs can be ignored because only two desks are involved

11. Fifth Level RAID: No Single Check Disk

While level 4 RAID achueved parallelism for reads, writes are still limited to one per group since every write must read and write the check disk The final level RAID distributes the data and check information across all the disks--including the check disks Figure 4 compares the location of check information in the sectors of disks for levels 4 and 5 RAIDs

The performance impact of this small change is large since RAID level 5 can support multuple individual writes per group For example, suppose in Figure 4 above we want to write sector 0 of disk 2 and sector 1 of disk 3 As shown on the left Figure 4, in RAID level 4 these writes must be sequentual since both sector 0 and sector 1 of disk 5 must be written However, as shown on the nght, in RAID level 5 the writes can proceed in parallel since a write to sector 0 of disk 2 still involves a write to disk 5 but a write to sector 1 of disk 3 involves a write to disk 4

These changes bnng RAID level 5 near the best of both worlds small read-modify-writes now perform close to the speed per disk of a level 1 RAID while keeping the large transfer performance per disk and high useful storage capacity pencentage of the RAID levels 3 and 4 Spreading the data across all disks even improves the performance of small reads, since there is one more disk per group that contains data Table VI summarizes the charactenstics of this RAID

Keeping in mind the caveats given earlier, a Level 5 RAID appears very attractive if you want to do just supercomputer applications, or just transaction processing when storage capacity is limited, or if you want to do both supercomputer applications and transaction processing

12. Discussion

Before concluding the paper, we wish to note a few more interestung points about RAIDs The first is that while the schemes for disk striping and party support were presented as if they were done by hardware, there is no necessity to do so We just give the method, and the decision between hardware and software solutions is strictly one of cost and benefit. For example, in cases where disk buffering is effectuve, there is no extra disks reads for level 5 small writes since the old data and old panty would be in main memory, so software would give the best performance as well as the least cost.

In this paper we have assumed the transfer unit is a multuple of the sector As the size of the smallest transfer unit grows larger than one

Check
Disk
s5 $\square$
(a) Check information for
Level 4 RAID for $G=4$ and
C=1 1 The sectors are shown
below the disks (The
checked areas indicate the
check information ) Writes
to s0 of disk 2 and sl of
disk 3 imply writes to so
and sl of disk 5 The
check disk (5) becomes the
write bottleneck.

Figure 4 Location of check information per sector for Level 4 RAID us. Level 5 RAID

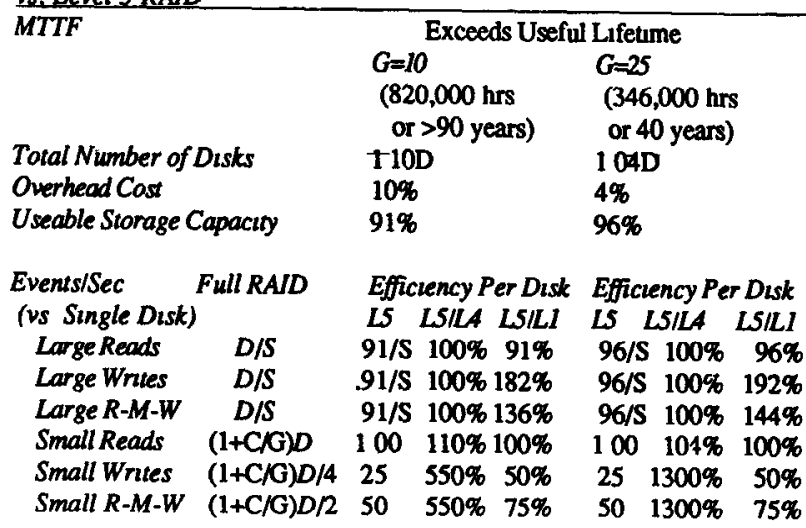

Table VI Characteristics of a Level 5 RAID The LSILA column gives the \% performance of $L S$ in terms of $L A$ and the $L / L I$ column gives at in terms of $L I$ ( $>100 \%$ means $L S$ is faster) Because reads can be spread over all disks, including what were check disks in level 4. all small I/Os improve by a factor of $1+C / G$ Small writes and $R-M-W$ s improve because they are no longer constrained by group size, getting the full disk bandwidth for the 4 IIO's associated with these accesses We again make the same assumptions as we made in Tables II and $V$ the slowdown for two related IIOs can be ignored because only two dusks are involved sector per dnve--such as a full track with an $1 / 0$ protocol that supports data returned out-of-order--then the performance of RAIDs improves significantly because of the full track buffer in every disk For example, if every disk begins transferring to its buffer as soon as it reaches the next sector, then $S$ may reduce to less than 1 since there would be virtually no rotauonal delay With transfer units the size of a track, it is not even clear if synchronizing the disks in a group improves RAID performance

This paper makes two separable points the advantages of building I/O systems from personal computer disks and the advantages of five different disk array organizations, independent of disks used in those array The later point starts with the traditıonal mirrored disks to achieve acceptable reliability, with each succeeding level improving

- the data rate, characterized by a small number of requests per second for massive amounts of sequential information (supercomputer applications), 
- the IIO rate, characterzed by a large number of read-modify-writes to a small amount of random information (transaction-processing),

- or the useable storage capacity, or possibly all three

Figure 5 shows the performance improvements per disk for each level RAID The highest performance per disk comes from either Level 1 or Level 5 In transaction-processing situations using no more than $50 \%$ of storage capacity, then the choice is murrored disks (Level 1) However, if the situation calls for using more than $50 \%$ of storage capacity, or for supercomputer applications, or for combined supercomputer applications and transaction processing, then Level 5 looks best Both the strength and weakness of Level 1 is that it duplicates data rather than calculating check information, for the duplicated data improves read performance but lowers capacity and wnte performance, while check data is useful only on a failure

Inspired by the space-tume product of paging studies [Denning 78], we propose a single figure of ment called the space-speed product the useable storage fraction times the efficiency per event Using this metric, Level 5 has an advantage over Level 1 of 17 for reads and 33 for wntes for $G=10$

Let us return to the first point, the advantages of bulding $I / O$ system from personal computer disks Compared to traditional Single Large Expensive Disks (SLED), Redundant Arrays of Inexpensive Disks (RAID) offer significant advantages for the same cost Table VII compares a level 5 RAID using 100 inexpensive data disks with a group size of 10 to the IBM 3380 As you can see, a level 5 RAID offers a factor of roughly 10 improvement in performance, reliability, and power consumption (and hence air conditioning costs) and a factor of 3 reduction in size over this SLED Table VII also compares a level 5 RAID using 10 inexpensive data disks with a group size of 10 to a Fujitsu M2361A "Super Eagle" In this comparison RAID offers roughly a factor of 5 improvement in performance, power consumption, and size with more than two orders of magnitude improvement in (calculated) reliability

RAID offers the further advantage of modular growth over SLED Rather than being limited to 7,500 MB per increase for $\$ 100,000$ as in the case of this model of IBM disk, RAIDs can grow at either the group size (1000 MB for $\$ 11,000)$ or, if partual groups are allowed, at the disk size (100 MB for $\$ 1,100$ ) The flip side of the coin is that RAID also makes sense in systems consıderably smaller than a SLED Small incremental costs also makes hot standby spares practucal to further reduce MTTR and thereby increase the MTTF of a large system For example, a 1000 disk level 5 RAID with a group size of 10 and a few standby spares could have a calculated MTTF of over 45 years

A final comment concerns the prospect of designing a complete transaction processing system from either a Level 1 or Level 5 RAID The drastically lower power per megabyte of inexpensive disks allows systems designers to consider battery backup for the whole disk array--the power needed for 110 PC disks is less than two Fujıtsu Super Eagles Another approach would be to use a few such disks to save the contents of battery backed-up main memory in the event of an extended power failure The smaller capacity of these disks also ties up less of the database during reconstruction, leading to higher avalability (Note that Level 5 ties up all the disks in a group in event of fallure while Level 1 only needs the single mirrored disk during reconstruction, giving Level 1 the edge in avarlability)

\section{Conclusion}

RAIDs offer a cost effectuve option to meet the challenge of exponentral growth in the processor and memory speeds We believe the size reduction of personal computer disks is a key to the success of disk arrays, just as Gordon Bell argues that the size reduction of microprocessors is a key to the success in muluprocessors [Bell 85] In both cases the smaller size simplifies the interconnection of the many components as well as packaging and cabling While large arrays of mainframe processors (or SLEDs) are possible, it is certainly easier to construct an array from the same number of microprocessors (or PC drives) Just as Bell coined the term "multı" to distunguish a multuprocessor made from microprocessors, we use the term "RAID" to identufy a disk array made from personal computer disks

With advantages in cost-performance, reliability, power consumption, and modular growth, we expect RAIDs to replace SLEDs in future I/O systems There are, however, several open issues that may bare on the practucality of RAIDs

- What is the umpact of a RAID on latency?

- What is the impact on MTTF calculations of non-exponential fallure assimptions for indrvidual disks?

- What will be the real lifetume of a RAID vs calculated MTTF using the independent fallure model?

- How would synchronized disks affect level 4 and 5 RAID performance?

- How does "slowdown" S actually behave? [Livny 87]

- How do defective sectors affect RAID?

- How do you schedule IIO to level 5 RAIDs to maximize write parallelusm?

- Is there locality of reference of disk accesses in transaction processing?

- Can information be automatically redistributed over 100 to 1000 disks to reduce contentron?

- Will disk controller design limit RAID performance?

- How should 100 to 1000 disks be constructed and phystcally connected to the processor?

- What is the impact of cabling on cost, performance, and reliability?

- Where should a RAID be connected to a CPU so as not to limit performance? Memory bus? I/O bus' Cache?

- Can a file system allow differ striping polucies for different files?

- What is the role of solid state disks and WORMs in a RAID?

- What is the umpact on RAID of "parallel access" disks (access to every surface under the read/write head in parallel)?
$\mathbf{Q}$ Large I/O $\mathrm{O}$ Small I/O $\mathbf{E}$ Capacity

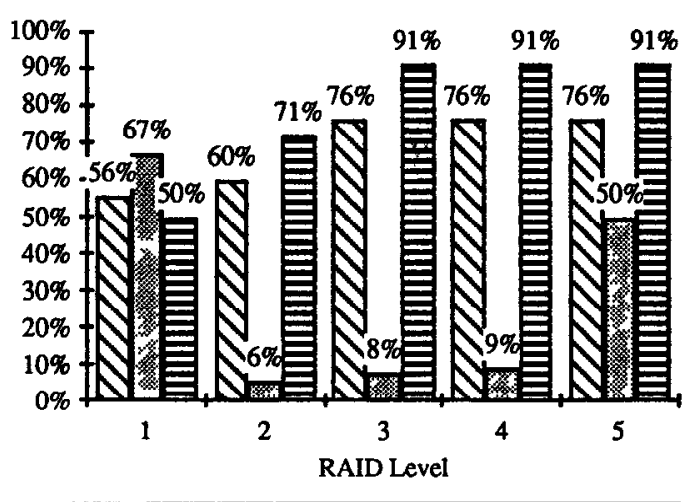

Figure 5 Plot of Large (Grouped) and Small (Individual) Read-Modify-Writes per second per disk and useable storage capacity for all five levels of RAID $(D=100, G=10)$ We assume a single $S$ factor uniformly for all levels with $S=13$ where it is needed
Charactersacs

(1)

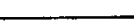

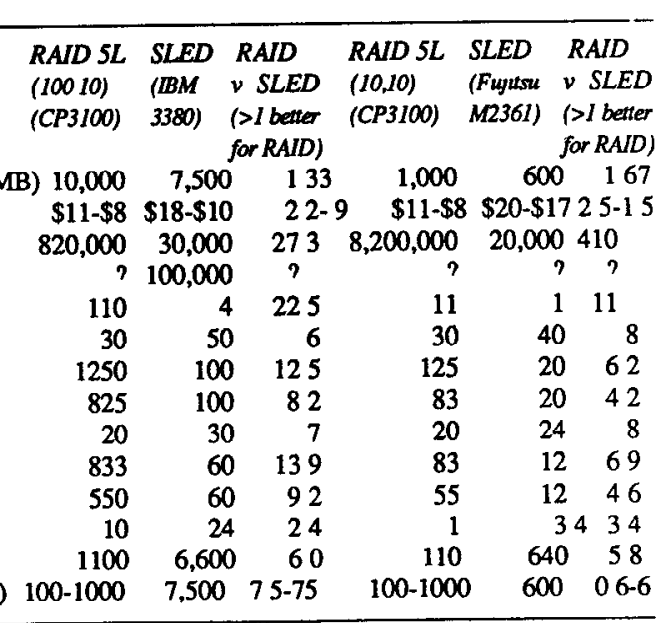

Formatted Data Capacity (MB) $10,000 \quad 7,500 \quad 133 \quad 1,000 \quad 600 \quad 167$

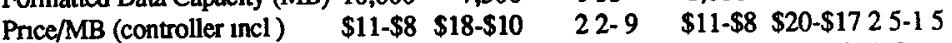
$820,000 \quad 30,000 \quad 273 \quad 8,200,000 \quad 20,000410$

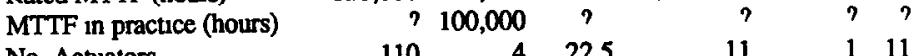
$\begin{array}{lrrrrr}\text { No Actuators } & 110 & 4 & 225 & 11 & 1 \\ \text { Max IO's/Actuator } & 30 & 50 & 6 & 30 & 40\end{array}$ $\begin{array}{lllllll}\text { Max Grouped RMW/box } & 1250 & 100 & 125 & 125 & 20 & 62\end{array}$ $\begin{array}{lrrrrrr}\text { Max Individual RMW/box } & 825 & 100 & 82 & 83 & 20 & 42\end{array}$ $\begin{array}{lrrrrrrr}\text { Typ I/O's/Actuator } & 20 & 30 & 7 & 20 & 24 & 89\end{array}$

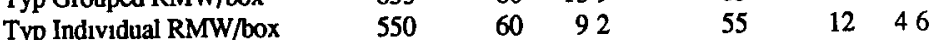
$\begin{array}{lrrrrrr}\text { Volume/Box (cubic feet) } & 10 & 24 & 24 & 1 & 34 & 34 \\ & 1100 & 6,600 & 60 & 110 & 640 & 58\end{array}$

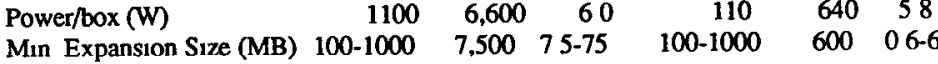

Table VII Comparison of IBM 3380 disk model AK4 to Level 5 RAID using 100 Conners \& Associates CP 3100 s disks and a group size of 10 and a comparison of the Fujtsu M2361A "Super Eagle" to a level 5 RAID using 10 inexpensive data disks with a group size of 10 Numbers greater than 1 in the comparison columns favor the RAID 


\section{Acknowledgements}

We wish to acknowledge the following people who participated in the discussions from which these ideas emerged Michael Stonebraker, John Ousterhour, Doug Johnson, Ken Lutz, Anapum Bhide, Gaetano Bonello Mark Hill, David Wood, and students in SPATS seminar offered at U C Berkeley in Fall 1987 We also wish to thank the following people who gave comments useful in the preparation of this paper Anapum Bhide, Pete Chen, Ron David, Dave Ditzel, Fred Douglis, Dieter Gawlick, Jim Gray, Mark Hill Doug Johnson, Joan Pendleton, Martin Schulze, and Hervé Touau This work was supported by the National Science Foundation under grant \# MIP-8715235

\section{Appendix Reliability Calculation}

Using probability theory we can calculate the MTTF Group $_{\text {We first }}$ assume independent and exponentual falure rates Our model uses a biased coin with the probability of heads being the probability that a second fallure will occur within the MTTR of a first fallure Since disk fallures are exponential

Probability(at least one of the remainung disks falling in MTTR) $=\left[1-\left(\mathrm{e}^{-\mathrm{MTTR} / \mathrm{MTTF}} \mathrm{Disk}\right)(\mathrm{G}+\mathrm{C}-1)\right]$

In all pracucal cases

$$
\text { MTTR } \ll \frac{\text { MTTF }_{D_{1 s k}}}{G+C}
$$

and since $\left(1-\mathrm{e}^{-\mathrm{X}}\right)$ is approximately $\mathrm{X}$ for $0<\mathrm{X} \ll 1$

Probability(at least one of the remaining disks falling in MTTR) $=\operatorname{MTTR}^{*}(\mathrm{G}+\mathrm{C}-1) / \mathrm{MTTF}_{\mathrm{D}_{\text {1sk }}}$

Then that on a disk failure we flip this coin

heads $\Rightarrow>$ a system crash, because a second fallure occurs before the first was reparred. Then

tails $\Rightarrow$ recover from error and contunue

MTTF $_{\text {Group }}=$ Expected[Tume between Falures]

* Expected[no of flips unul first heads]

Expected[Tume between Falures]

$=$

Probability(heads)

$=\frac{\text { MTTF }_{\text {Disk }}}{(G+C)^{*}\left(M_{T T R}^{*}(G+C-1) / M_{T T F_{1 s k}}\right)}$

$$
\text { MTTF }_{\text {Group }}=\frac{\left(\text { MTTF }_{\text {Disk }}\right)^{2}}{(G+C)^{*}(G+C-1)^{*} \text { MTTR }}
$$

Group farlure is not precisely exponental in our model, but we have validated this simplifying assumption for pracucal cases of MTTR « MTTF/ $(\mathrm{G}+\mathrm{C})$ This makes the MTIF of the whole system Just MTTF $_{\text {Group }}$ divided by the number of groups, $n_{G}$

\section{References}

[Bell \$4] C G Bell, "The Minı and Micro Industries," IEEE Computer Vol 17 No 10 (October 1984), pp 14-30

[Jo) 85 B Jos presentation at ISSCC '85 panel session, Feb 1985

[Siewioreh 82] D P Sicu 1oreh, C G Bell, and A Newell, Computer Strue tures Prinu iples and Examples, $\mathrm{p} 46$

[Moore 75] GE Moore, "Progress in Digital Integrated Electronics," Proc ICEE Digital Integrated Electronu, Device Meettng, (1975), p 11

[M)ers 86] G J Mycrs A Y C Yu, and D I. House, "Microprocessor Technology Trends " Proc ILLE, Vol 74, no 12, (December 1986), pp 1605-1622

[Garcia 84] H Garcia Molina, R Cullingford, P Honeyman, R Lipton, "The Case for Massive Memory," Technical Report 326, Dept of EE and CS, Prunceton Unıv, May 1984

[Myers 86] W Myers, "The Compeutuveness of the Unuted States Disk Industry," IEEE Computer, Vol 19, No 11 (January 1986), pp 85-90

[Frank 87] PD Frank, "Advances in Head Technology," presentation at Challenges in Disk Technology Short Course, Instutute for Information Storage Technology, Santa Clara University, Santa Clara, Calıfornia, December 15-17, 1987

[Stevens 81] L D Stevens, "The Evolution of Magnetuc Storage," IBM Journal of Research and Development, Vol 25, No 5, Sept 1981, pp 663-675

[Harker 81] J M Harker et al, "A Quarter Century of Disk File Innovation," lbıd, pp 677-689

[Amdahl 67] G M Amdahl, "Valıdity of the single processor approach to achievng large scale computung capabulites," Proceedings AFIPS 1967 Spring Joint Computer Conference Vol 30 (Atlantic City, New Jersey Apnl 1967), pp 483-485

[Boral 83] $\mathrm{H}$ Boral and $\mathrm{DJ}$ DeWitt, "Database Machines An Ideas Whose Tume Has Passed? A Critique of the Future of Database Machines," Proc International Conf on Database Machines, Edited by H -O Lelich and M Misskoff, Springer-Verlag, Berlin, 1983

[IBM 87] "IBM 3380 Direct Access Storage Introduction," IBM GC 26-4491-0, September 1987

[Gawlick 87] D Gawlick, private communication, Nov , 1987

[Fujtsu 87] "M2361A Mini-Disk Drive Engineenng Specifications," (revised) Feb , 1987, B03P-4825-0001A

[Adaptec 87] AIC-6250, IC Product Gulde, Adaptec, stock \# DB0003-00 rev $B, 1987$, p 46

[Livny 87] Livny, M, S Khoshafian, H Boral, "Multı-disk management algorithms," Proc of ACM SIGMETRICS, May 1987

[Kım 86] M Y Kım, "Synchronized disk interleaving," IEEE Trans on Computers, vol C-35, no 11, Nov 1986

[Salem 86] K Salem and Garcia-Molına, H, "Disk Striping," IEEE 1986 Int Conf on Data Engineering, 1986

[Bitton 88] D Bitton and J Gray, "Disk Shadowing," in press, 1988

[Kurzweil 88] F Kurzwell, "Small Disk Arrays - The Emerging Approach to High Performance," presentation at Spring COMPCON 88, March 1, 1988, San Francisco, CA

[Hamming 50] R W Hamming, "Error Detecung and Correctung Codes," The Bell System Technical Journal, Vol XXVI, No 2 (Apni 1950), pp 147-160

[Hillis 87] D Hillss, private communication, October, 1987

[Park 86] A Park and K Balasubramantan, "Providing Fault Tolerance in Parallel Secondary Storage Systems," Department of Computer Science, Princeton University, CS-TR-057-86, Nov 7, 1986

[Maginnis 87] N B Maginnis, "Store More, Spend Less Mid-range Options Abound," Compuserworld, Nov 16, 1987, p 71

[Denning 78] P.J Denning and D F Slutz, "Generalized Working Sets for Segment Reference Strings," CACM, vol 21, no 9, (Sept. 1978) pp $750-759$

[Bell 85] Bell, C G, "Multıs a new class of multıprocessor computers," Science, vol. 228 (April 26, 1985) 462-467 